\title{
Diferentes aplicaciones del vídeo como herramienta docente en un Máster virtual
}

\author{
Delgado García, Ana María ${ }^{1}$; Rovira Ferrer, Irene ${ }^{2}$; Oliver Cuello, Rafael ${ }^{3}$ \\ ${ }^{1}$ Catedrática de Derecho Financiero y Tributario de la Universitat Oberta \\ de Catalunya, adelgadoga@uoc.edu \\ ${ }^{2}$ Profesora agregada de Derecho Financiero y Tributario de la Universitat \\ Oberta de Catalunya, iroviraf@uoc.edu \\ ${ }^{3}$ Catedrático de Derecho Financiero y Tributario de ESERP Business \& \\ LawSchool,roliver@uoc.edu
}

\begin{abstract}
Some of the elements that have been most transformed after the installation of the European Higher Education Area (EHEA) and the generalization of Information and Communication Technologies (ICT) have been the learning resources, a point at which the videos of a teaching nature is configured as a tool of high potential. For example, the results in the Master's Degree in Taxation (MUF) of the Open University of Catalonia (UOC), constitute the proposal of the different uses of the same the main objective of this work: the use of video by the part of the students the task of carrying out the tasks, the use of contents related to the content of some subjects to consolidate them and the integral formation and the elaboration of the own videos by the teaching team to facilitate the learning. In the three cases, there have been few drawbacks (especially those focused on the work that is supposed to be created and that has been compensated with its possible reuse) and many advantages for both students and teachers, so it is clearly They raise As work tools with a suitable potential for any degree.
\end{abstract}

Keywords: Master's degree, ICT, video, taxation

\footnotetext{
Resumen

Unos de los elementos que más se han visto transformados tras la instauración del Espacio Europeo de Educación Superior (EEES) y la generalización de las Tecnologías de la Información y la Comunicación (TIC) han sido los recursos de aprendizaje, punto en el que los vídeos de carácter docente se configuran como una herramienta de elevado potencial. Por ello, conscientes de su relevancia, la apuesta por su implementación ha sido uno de los principales objetivos en el Máster Universitario de Fiscalidad (MUF) de la Universitat Oberta de Catalunya (UOC),
} 
constituyendo la propuesta de tres usos distintos de los mismos el principal objetivo del presente trabajo: la utilización del vídeo por parte de los estudiantes para garantizar la adquisición de determinadas competencias, la utilización de vídeos externos relacionados con el contenido de algunas asignaturas para consolidar contenidos específicos y reforzar la formación integral y la elaboración de vídeos propios por parte del equipo docente para facilitar el aprendizaje. En los tres casos, han sido pocos los inconvenientes que plantean (especialmente focalizados en el trabajo que supone su creación pero que se ve compensado con su posible reutilización) y muchas las ventajas tanto para los estudiantes como los profesores, por lo que claramente se alzan como herramientas docentes con un potencial idóneo para cualquier titulación.

Palabras clave: Máster universitario, TIC, vídeo, fiscalidad

\section{Introducción}

Desde la instauración del EEES, el proceso de aprendizaje ha dejado atrás la impartición de clases magistrales para pasar a fundamentarse en la adquisición de competencias, definidas como "el conjunto de conocimientos, saberes, actitudes y habilidades para realizar con efectividad determinadas acciones" (González Lozada \& Muñoz Catalán, 2010).

Así, en el caso de los Grados y Másteres en Derecho, el diseño del programa debe trascender de la mera transmisión y memorización de contenidos, en el sentido de que debe garantizar la adquisición de las "capacidades esenciales que debe desarrollar el estudiante de Derecho para ser un verdadero jurista” (Rivera Ayala, 2014).

Por consiguiente, a la hora de desarrollar el pertinente procedimiento de aprendizaje, resulta necesario que hoy se sitúe a los estudiantes como los principales protagonistas de su formación, de modo que la labor fundamental del profesorado ha pasado a recaer en dos grandes cometidos: por un lado, el diseño y planificación de recursos y actividades orientadas a la adquisición de las debidas competencias que integran los programas (donde resulta crucial la potenciación de la formación práctica) y, por otro, la ofrecimiento de la guía, el apoyo y el estímulo necesario para que los estudiantes puedan lograrlo con éxito.

Y dentro de este contexto, la utilización de las TIC abre un enorme abanico de posibilidades sin precedentes, de modo que, gracias a las mismas y a la reconfiguración del proceso metodológico tradicional, han aparecido nuevas formas de aprender, estudiar y evaluar partiendo de nuevos elementos y recursos de aprendizaje (Cabero Almenar, 1996).

Así pues, la constante innovación basada en el uso de las TIC resulta un reto ineludible en toda la enseñanza superior, lo cual viene siendo un objetivo permanente en el Máster 
Universitario de Fiscalidad de la UOC (dado que, además, la relevancia de la potencialidad de las nuevas herramientas y recursos se ve maximizada en la formación no presencial).

Por ello, y conscientes de las grandes ventajas que puede aportar su utilización, decidimos en este caso apostar por la incorporación de vídeos como nuevas herramientas docentes, los cuales, suponen la evolución natural de la grabación de clases completas aunque difiriendo del todo por lo que respecta a su duración, soporte, metodología, filosofía y formato (Letón, García-Saiz, Fernández-Vindel, Boticario, Luque, Rodríguez-Ascaso, Hernández-del-Olmo, Sarro, San Cristóbal, Rivas, Lz. de Sosoaga Torija \& Quintana-Frías, 2011).

Sin embargo, tanto las características como las finalidades de los vídeos que se pueden implementar en la educación superior son de muy variada índole, por lo que se ha considerado de interés destinar el presente trabajo a explicar la incorporación de tres usos diferenciados de los mismos por los que se ha optado en el ámbito del Máster Universitario de Fiscalidad.

\section{Objetivos}

El principal objetivo en el que se enmarca el esfuerzo por la constante innovación docente es la mejora de la calidad de nuestra enseñanza, aunque, en este caso, una vez decidida la apuesta por la incorporación de vídeos docentes, pretendimos focalizarnos en conseguir cuatro grandes retos: en primer lugar, facilitar el aprendizaje de nuestros estudiantes; en segundo lugar, maximizar la adquisición de las competencias profesionalizadoras (y más considerando la formación avanzada y dirigida a especialidades concretas que define los Másteres); en tercer lugar, salvar las barreras que presenta la enseñanza no presencial a la hora de garantizar el trabajo de las competencias basadas en la oralidad; y, en cuarto lugar, mejorar la satisfacción en nuestros recursos de aprendizaje.

Por ello, el primer objetivo que nos planteamos fue una breve revisión teórica sobre la utilización docente de los vídeos, a partir del cual nos marcamos como segundo objetivo el estudio de cómo podíamos incorporarlos en el Máster Universitario de Fiscalidad para dar respuesta a las concretas finalidades perseguidas.

Seguidamente, una vez tuvimos claro cómo podíamos utilizarlos, el tercer objetivo fue la especificación en cada caso de qué vídeos utilizar para la maximización de los resultados perseguidos, así como la detección de aquellas asignaturas en las que su incorporación ofrecía un mayor potencial.

Y finalmente, después de detallar su incorporación, el cuarto gran objetivo era la valoración de la iniciativa docente, destacando los aciertos y las dificultades encontradas y apuntando posibles propuestas de mejora y recomendaciones con base en la experiencia constatada. 


\section{Desarrollo de la innovación}

El primer paso que debíamos realizar a la hora de implementar los vídeos docentes en MUF era un breve estudio teórico acerca de los mismos, analizando, en especial, su relevancia en los Grados y Másteres de Derecho.

\subsection{Los vídeos como herramientas docentes}

El estudio de los vídeos en calidad de herramientas docentes ha abierto un nuevo campo de investigación en el ámbito educativo, ya que son múltiples características y las finalidades que pueden entrañar. A modo de ejemplo, Letón, Gómez del río, Quintana-frías \& Molanes-López distinguen hasta 252 tipos de lo que ellos denominan "distintas modalidades de grabación", distinguiendo 4 categorías en función de su duración (los micro-vídeos -menos de 5 minutos-, los mini-vídeos -entre 5 y 10-, los maxi-vídeos -entre 10 y 25- y los vídeos -más de 25-), 9 categorías considerando su diseño (los propiamente docentes, los minimalistas, los maximalistas, los multimedia, los tutoriales, los de notas, los de conferencias, los de entrevistas y los de reportajes), y 7 categorías con base en su objetivo (los modulares, los magistrales, los magistrales con interacción, los promocionales, los motivadores, los sociales y los de entretenimiento) (Letón, Gómez del río, Quintanafrías \& Molanes-López, 2012).

No obstante, como resalta MEDINA, los beneficios que presentan son manifiestos en todos los casos, ya que permiten que el estudiante controle su reproducción; suponen una ventaja sobre los apuntes en tanto que contienen en audio las explicaciones del profesor; constituyen un nuevo material con el que cuentan los estudiantes y que, en consecuencia, puede facilitar y enriquecer su estudio; permiten crear material adicional posibilitando un aprendizaje más completo; y, al tratarse de un material multimedia, pueden hacer más llevadera la tarea de estudio centrada esencialmente en la lectura de apuntes y libros (Medina, 2008).

Además, el hecho de que tales vídeos estén disponibles online, permite que los estudiantes puedan acceder a los mismos en cualquier momento y desde cualquier lugar con conexión a Internet (ya sea con un ordenador, un portátil, una tableta o un teléfono móvil), sin olvidar que, a pesar de que su elaboración implique una importante labor por parte del profesorado, su contenido puede reutilizarse y probablemente disminuirá el número de preguntas y errores que se generen durante el estudio.

Pero es de suma relevancia destacar que la gran potencialidad del vídeo como recurso de aprendizaje va mucho más allá de los "mini-vídeos docentes" (concepto creado por Letón, Durbán, d'Auria y Lee y definido como la simbiosis de la pizarra tradicional, las transparencias (diapositivas) y los vídeos de clase completas, teniendo una duración aproximada de 5 a 10 minutos (Letón, Durbán, D’Auria \& Lee, 2009), ya que, aparte de poderse utilizar vídeos creados por organismos oficiales y no sólo los elaborados por los profesores para consolidar determinados conceptos, permiten fomentar la formación 
práctica a través de tutoriales de portales o aplicaciones en aquellas titulaciones cuyo uso requiere la pertinente práctica profesional (lo cual ocurre en gran medida en el ámbito de la fiscalidad considerando que la Administración tributaria española constituye un referente de ámbito mundial en la incorporación de las TIC).

Y en la misma línea procede resaltar que, especialmente en la formación no presencial, su elaboración por parte de los estudiantes resulta un recurso prácticamente imprescindible para poder trabajar las competencias relacionadas con la oralidad que contiene todo Grado o Máster jurídico, al mismo tiempo que ello les obliga a dotarlos de la formalidad necesaria simulando una situación que acabará formando parte de su práctica profesional.

Así pues, una vez sentadas las características principales de los vídeos docentes y partiendo de nuestros objetivos iniciales (facilitar el aprendizaje de nuestros estudiantes, maximizar la adquisición de las competencias profesionalizadoras, salvar las barreras que presenta la enseñanza no presencial a la hora de garantizar el trabajo de las competencias basadas en la oralidad y mejorar de la satisfacción de nuestros recursos de aprendizaje), vimos que, aunque de diferentes formas, podíamos dividir la potencialidad de su incorporación en dos finalidades básicas: garantizar el íntegro trabajo de competencias que conforman nuestro programa y mejorar la adquisición de las mismas.

\subsection{La utilización del vídeo docente para garantizar la íntegra adquisición de competencias del Máster Universitario de Fiscalidad}

A pesar de que actualmente la adquisición de competencias que deben garantizar los programas va mucho más allá de la mera transmisión de conocimientos, es evidente que la correcta comprensión e integración de los mismos resulta también imprescindible para lograr los resultados de aprendizaje perseguidos, y en este punto, como se ha puesto de manifiesto, los vídeos docentes se configuran como un elemento de alto potencial.

Por lo que respecta a las competencias transversales del Máster Universitario de Fiscalidad, estamos obligados a garantizar el trabajo de las siguientes: la utilización a un nivel avanzado de Internet, bases de datos jurídico-tributarias y otras herramientas informáticas propias del ámbito fiscal; la búsqueda de información, documentación y fuentes de referencia tributaria a través de las TIC; la capacidad para interpretar textos de naturaleza tributaria en inglés; la capacidad para aplicar el conocimiento tributario a la práctica profesional, detectando y resolviendo situaciones problemáticas y complejas; la toma de decisiones en el ámbito de la asesoría empresarial y personal o de la aplicación tributaria en las Administraciones públicas, de acuerdo con la deontología y la ética profesional exigibles en esta materia; el aprendizaje autónomo y permanente para una correcta reacción y adaptación ante la cambiante y completa realidad fiscal en un entorno globalizado y de pluralismo jurídico; y el trabajo en equipo y capacidad de liderazgo en el ámbito tributario, planificando y gestionando el tiempo de forma adecuada.

Y por su parte, en relación con las competencias específicas, debemos velar por la adquisición de las siguientes: la consulta y utilización correcta de las fuentes tributarias (legales, jurisprudenciales y doctrinales); la capacidad de análisis, síntesis y de relación de 
las normas y figuras tributarias; la capacidad de interpretación y comparación de datos y fuentes de naturaleza tributaria en un entorno complejo, cambiante y global; el diseño de estrategias de actuación en el campo tributario para un asesoramiento fiscal óptimo, de acuerdo con los principios del ordenamiento tributario y la finalidad de las normas fiscales; la capacidad para la elaboración y redacción de informes y dictámenes, recursos, declaraciones, formularios y otra documentación en el ámbito tributario; el análisis crítico del ordenamiento tributario y de su aplicación; y la negociación y argumentación en el entorno profesional tributario.

\section{Resultados}

Al tratarse de un Máster completamente on line, un tema que nos preocupaba era cómo trabajar la competencia de expresión/argumentación oral. Precisamente, el video nos permite valorar cómo desarrolla esta competencia el estudiante. Por este motivo, la primera asignatura en la que se introdujo el vídeo, hace tres años, para asegurar que se trabajaba la oralidad en dichas competencias fue en el Trabajo Final de Máster (TFM). En concreto, los estudiantes deben grabar por cualquier medio (webcam de su ordenador, móvil...) un video, de entre 5 y 10 minutos, presentando su TFM y lo deben subir a una aplicación diseñada por la UOC, denominada langblog, que permite también la grabación de videos. Desde esta aplicación el profesor puede visualizar el video y proceder a la valoración de las competencias.

Una de las dificultades que se observaron es que los estudiantes no están demasiado habituados a grabarse en video. De ahí que decidimos elaborar un tutorial para ayudarles. Así, el profesor de la asignatura a través de otro video elaborado por él les da pautas sobre cómo hacerlo y lo cierto es que desde que se puso a disposición de los estudiantes dicho video, los resultados de los videos elaborados por los estudiantes mejoraron y también la satisfacción de los estudiantes con la asignatura.

Tras esta experiencia, y dado que en el Practicum también tenían que poner en práctica los estudiantes la competencia de la oralidad, decidimos hace dos años que en la última de las tareas que entregan los estudiantes (un informe sobre los asuntos tratados para el asesor fiscal para el que han trabajado) hicieran un resumen de dicho informe a través de un video, detallando los aspectos más destacados de los asuntos y los problemas a los que han tenido que enfrentarse. Nuevamente, la valoración de los estudiantes de esta metodología ha sido positiva, pues les obliga a poner en práctica no sólo la competencia de la oralidad, sino otras como la argumentación, la capacidad de síntesis y de crítica.

A la vista de los buenos resultados de estas dos iniciativas y que se plasmaron en el aumento de 10 puntos porcentuales en las encuestas de satisfacción que realizaron los estudiantes tanto en el Practicum (Encuesta de satisfacción del Máster Universitario de Fiscalidad del curso 2017-18) como en el Trabajo Final de Máster, hemos decidido incorporar este curso académico en ocho asignaturas diversos vídeos externos elaborados por órganos oficiales en los que se profundiza con la explicación de determinados conceptos o contenidos de algunas asignaturas, como es el caso de las notificaciones 
electrónicas que se trabajan en la asignatura de Procedimientos de gestión e inspección tributaria (donde se ha incorporado un vídeo sobre las mismas elaborado por la Administración Estatal de la Administración Tributaria (AEAT) https://youtu.be/zYAvKiVCQg8).

Sin embargo, considerando la importancia de formar a los estudiantes de la forma más íntegra y cercana posible a su futura práctica profesional y la potenciación de la adquisición de algunas de las competencias señaladas (como la utilización a un nivel avanzado de Internet, bases de datos jurídico-tributarias y otras herramientas informáticas propias del ámbito fiscal), también se realizó un importante esfuerzo para integrar vídeos elaborados por la Administración tributaria española en los que se explica tanto la navegación por la Sede electrónica de la AEAT (https://youtu.be/Fe49117BQRY) como la realización de trámites específicos (como puede ser la elaboración y presentación de determinadas autoliquidaciones, la solicitud de un certificado tributario o la consulta de un expediente electrónico), y es que no sólo se encuentran disponibles a través de Internet la práctica totalidad de trámites que debemos hacer los juristas con la Administración tributaria, sino que en muchos casos resulta incluso obligatorio.

Todos estos videos externos tienen la característica de que son videos breves y están en acceso abierto, en línea con la política de nuestra Universidad de promocionar el conocimiento abierto.

Finalmente, otro proyecto que deseamos implementar en el curso académico 2019-2020 es la de elaborar unos videos en el Practicum, con la participación del profesorado de la asignatura, en los que se recrea una reunión de un asesor fiscal con su cliente y de un inspector de Hacienda con un contribuyente. El motivo que nos guía en este caso a acudir al video es que el Practicum constituye una piedra angular del procedimiento de aprendizaje para la completa adquisición de las diferentes competencias del Máster -tanto por su finalidad de poner en práctica todos los conocimientos y habilidades trabajados a lo largo de la titulación como por permitir su íntegra evaluación- y la importancia de la formación práctica y lo más cercana posible a la práctica profesional.

\section{Conclusiones}

El video, como recurso docente, sirve para potenciar la formación práctica de los estudiantes, pues constituye una pieza clave para el correcto desarrollo de las diferentes competencias profesionalizadoras, entre las que destacan las relacionadas con la oralidad, negociación, capacidad crítica y capacidad de síntesis. Además, el video facilita la comprensión de conceptos no siempre fáciles de entender, pues la fiscalidad es una materia compleja, inabarcable y sometida a un constante proceso de mutabilidad. Y, en este contexto, contribuye a construir un conocimiento significativo, puesto que con un video se aprovecha el potencial comunicativo de las imágenes conjugado con los sonidos, lo cual, a su vez, permite conseguir una idea más real de los conceptos. 
Por otro lado, la introducción de recursos audiovisuales, permite avanzar en la diversificación tanto del formato de los distintos recursos docentes como de las fuentes de su procedencia y enriquece el aprendizaje.

Los videos por los que hemos apostado en el Máster Universitario de Fiscalidad son tanto videos grabados por los propios estudiantes como videos puestos a disposición de los mismos por parte del equipo docente (videos grabados por el propio equipo docente o bien videos externos). En todos los casos, se trata de videos de duración breve y en el caso de los videos externos son videos oficiales de instituciones especializadas en el ámbito fiscal y de acceso abierto.

La satisfacción de los estudiantes ha mejorado tras la implementación de los videos; en efecto, las encuestas de satisfacción evidenciaron un 10\% de incremento de satisfacción en el semestre académico en que se implementaron los videos. Además, se observó una mayor participación en el aula, pues el video incrementa la motivación y el interés de los estudiantes por la materia, puesto que más allá de transmitir información, más o menos exhaustiva y sistematizada, sobre un tema concreto, persigue suscitar interrogantes, identificar problemas, despertar el interés $y$, en definitiva, generar una dinámica participativa.

No obstante, debe destacarse que la dedicación del profesor se ha incrementado considerablemente no sólo por la tarea de seleccionar y gestionar los videos externos para que estén disponibles para los estudiantes en las aulas, sino también por la tarea de visionar los videos que graban los estudiantes en el caso de las asignaturas de Practicum y de Trabajo Final de Máster.

\section{Referencias}

CABERO ALMENAR, J. (1996). "Nuevas tecnologías, comunicación y educación". Revista Electrónica de Tecnología Educativa (EDUTEC) (1).

GONZÁLEZ LOZADA, S., \& MUÑOZ CATALÁN, E. (2010). “Análisis de las competencias de los estudiantes de Derecho en los nuevos planes de estudio de Andalucía en el EEES". Revista de Educación (12).

LETÓN, E., DURBÁN, M., D’AURIA, B. \& LEE, D.-J. (2009). “Self learning mini-vídeos through Internet and mobile telephones: a help to the student in the Bologna process". En Proceedings of EDULEARN09 Conference. 6th-8th July 2009, Barcelona. Barcelona: International Academy of Technology, Education and Development.

LETÓN, E., GARCÍA-SAIZ, T., FERNÁNDEZ-VINDEL, J.M., BOTICARIO, J.G., LUQUE, M., RODRIGUEZ-ASCASO, A., HERNÁNDEZ-DEL-OLMO, F., SARRO, L.M., SAN CRISTÓBAL, E., RIVAS, A., LZ. DE SOSOAGA TORIJA, A., \& QUINTANA-FRÍAS, I. (2011). "Mini-vídeos docentes modulares con pizarra electrónica". En AAVV IV Jornadas de redes de investigación en innovación docente. Madrid: UNED. 
LETÓN, E., GÓMEZ DEL RÍO, I., QUINTANA-FRÍAS, I., \& MOLANES-LÓPEZ, E.M. (2012). "Clasificación de las distintas modalidades de grabación y su relación con los minivídeos docentes modulares". En AAVV Actas del XVII Congreso Internacional de Tecnologías para la Educación y el Conocimiento. Madrid: UNED.

MEDINA MOLINA, J. (2008). "Un método para la generación de vídeos docentes". RevistaRect@, 16 (1).

RIVERA AYALA, L.A. (2014). "Cinco competencias genéricas clave en los estudios de Derecho”. Revista de Educación y Derecho (11). 